\title{
Lidil
}

Revue de linguistique et de didactique des langues

\section{Interprétation et dialogue chez les enfants et quelques autres}

FRANçOIS Frédéric, 2005, Lyon, ENS éditions, 267 p.

\section{Claude Le Manchec}

\section{(2) OpenEdition}

\section{Journals}

Édition électronique

URL : http://journals.openedition.org/lidil/72

DOI : $10.4000 /$ lidil.72

ISSN : 1960-6052

Éditeur

UGA Éditions/Université Grenoble Alpes

Édition imprimée

Date de publication : 1 juin 2006

Pagination : 171-178

ISBN : 2-914176-14-7

ISSN : 1146-6480

Référence électronique

Claude Le Manchec, «Interprétation et dialogue chez les enfants et quelques autres », Lidil [En ligne], 33 | 2006, mis en ligne le 01 octobre 2007, consulté le 21 septembre 2020. URL : http:// journals.openedition.org/lidil/72 ; DOI : https://doi.org/10.4000/lidil.72

Ce document a été généré automatiquement le 21 septembre 2020.

(c) Lidil 


\title{
Interprétation et dialogue chez les enfants et quelques autres
}

FRANÇOIS Frédéric, 2005, Lyon, ENS éditions, 267 p.

\author{
Claude Le Manchec
}

1 La difficulté de cette note de lecture sera d'essayer de ressaisir, à partir d'articles divers, publiés dans des contextes et à des époques différentes, le mouvement d'une pensée par ailleurs difficilement condensable en un discours de reprise. Notre ambition ne peut être que modeste, étant donné la densité d'un propos qui s'adresse autant aux linguistes qu'aux philosophes ou aux pédagogues. Pour y parvenir, il nous faudra parfois, en outre, être infidèle à l'ordre de présentation de ces onze articles répartis en trois grandes parties, et précédés d'une longue introduction qui, par son envergure, ouvre nombre de perspectives nouvelles en linguistique.

2 Dans la première partie, F. François tout en serrant de plus près certaines notions (l'interprétation, le discours notamment) replace ses propres recherches dans un cadre théorique où dominent deux noms, Bakhtine et Vygotski. En effet, selon lui, un dialogue inégal lie l'enfant à l'adulte mais ce dialogue débouche sur des mouvements discursifs qui restent à analyser aussi finement que possible en considérant l'évolution langagière de l'enfant à l'aide notamment du concept de dialogisme emprunté à Bakhtine ou à l'aide de la notion de zone de proche développement, empruntée à Vygotski.

Dans la deuxième partie de l'ouvrage, F. François souligne, en prenant appui sur plusieurs corpus, l'irréductibilité du langage de l'enfant à celui de l'adulte. Le langage de l'enfant, prévient-il, n'est pas seulement langage en mouvement vers celui de l'adulte. En réalité, une raison ludique s'y exerce, qui fait partie du fonctionnement même du langage.

Dans la même perspective - c'est l'objet de la troisième partie -, il est possible de mieux saisir la réalité du dialogue patient-psychiatre comme on l'a fait de celle du dialogue enfant-adulte. Le discours du patient peut être vu comme mouvement par rapport au discours de l'autre. De même que l'enfant n'apparaît pas forcément «en dessous " (démuni) par rapport à l'adulte, le discours pathologique n'est pas réductible aux 
théorisations unifiantes, généralement d'orientation psychanalytique, qui l'ont souvent pris pour objet d'étude spécifique.

5 Tout au long de la première partie de l'ouvrage, François ne cesse de questionner le discours scientifique qui s'est intéressé au développement du langage de l'enfant. Plusieurs mises en doute malmènent sérieusement certaines vérités autour desquelles les sciences du langage se sont constituées. F. François souligne ainsi à plusieurs reprises (p. 54, $79 \mathrm{sq}$.) les effets réducteurs du raisonnement scientifique lorsque, par exemple, il isole une structure de la langue d'un côté et une compétence communicative, de l'autre, alors que l'enfant va non pas des structures de la langue vers la parole mais des énoncés des autres aux énoncés de soi. De même, qu'est-ce qu'on gagne mais aussi qu'est-ce qu'on perd quand, par exemple, dans une perspective fonctionnaliste, on définit le langage par la fonction de communication alors qu'on peut communiquer autrement qu'avec le langage et qu'on ne fait pas que communiquer grâce au langage? Autre mise en doute : que fait-on lorsqu'on tente d'évaluer ou même seulement d'expliquer le développement du langage de l'enfant? sur quels modèles de la science s'appuie-t-on implicitement ? comment l'objet «scientifique » est-il découpé et que valent ces découpages (phonologie, syntaxe, lexique...) ? qu'en est-il de l'analyse de l'oral sachant que la linguistique a partie liée avec l'existence même de la langue écrite qui a donné forme aux lettres, mots, phrases (p. 53 sq.) ? qu'est-ce qui est ainsi sous-estimé ou simplement perdu de vue?

6 Pour avoir peu interrogé ces découpages, les spécialistes sont soit victimes d'effets de grossissement, soit passent littéralement à côté, par exemple, des liens qui unissent certaines évolutions lexicales et syntaxiques, d'une part, et l'expérience même du sujet, d'autre part. Or, comme le rappelle plusieurs fois François, le développement du langage est enté sur autre chose que lui-même. Le dialogue verbal et le dialogue nonverbal sont étroitement imbriqués comme on le voit dans le cas de la référence par déictique où l'orientation mutuelle du regard et le geste de monstration ont une grande importance. Le langage fait signe finalement dans son intégration à autre chose que lui et ce sont les enchaînements de message qui donnent sens au fait de raconter, d'argumenter...

7 Cette mise en doute de quelques axiomes fondateurs des sciences du langage conduit François à resituer les enjeux du dialogue enfant-adulte en soulignant l'importance de la notion d'interprétation dans le domaine de l'analyse du discours. De quoi est fait en effet le discours, notamment dans la communication enfant-adulte? Sur un thème commun, répond-il, qui se modifie au fur et à mesure du discours. Son attention se porte sur les mélanges des genres de discours mais aussi sur des procédures de déplacement-modification de ce même discours sous forme de changements d'accent, de changements de genres (narration, glose...), de modalisations, de changements de «mondes» (celui de l'expérience, celui du discours collectif reconnu...) ou encore de «remontées métadiscursives »... Ces changements correspondent au travail de l'interprétation entendue comme création d'un espace discursif où on peut mettre ensemble ce qui d'habitude ne va pas ensemble (du réel et de la fiction, par exemple, dans le cas du récit). Le sens se fabrique in situ en quelque sorte par les différences ("dénivellations») entre les interlocuteurs. Se met en place une communauté interprétative faite de reprises-modifications du discours de l'autre, où ce qui fait sens, ce sont non pas les énoncés eux-mêmes mais leur mouvement par rapport à ce qui précède. L'attention doit donc se porter sur les ajouts, reprises, et autres commentaires 
que François appelle, à la suite de Bakhtine, la compréhension-responsive au cœur du dialogue ordinaire.

8 Ceci posé - et ce sera l'objet de la deuxième partie de l'ouvrage - il reste à analyser de plus près le contenu même de ces mouvements discursifs dans le dialogue enfantadulte et d'en tirer quelques conséquences. Un énoncé se construit par reprises, modifications, commentaires, détournements du discours de l'autre, d'où l'importance de l'étude des tours de parole. Une étude d'un dialogue entre deux petites filles (p. 54 sq.) permet de mettre au jour des différences de fonctionnement entre les deux enfants. On s'intéressera dès lors à leur capacité distincte à changer de points de vue sur un thème commun de conversation. Deux mouvements différents, deux séries d'éléments jouent finalement en combinaison :

9 1) Le premier peut être rattaché à ce que les linguistes du discours appellent cohérence référentielle, cohésion linguistique lexicale et grammaticale, enchaînements codés : c'est le problème de la « communauté » discursive qui lie les interlocuteurs.

10 2) Le deuxième, étudié précisément dans une séquence conversationnelle (ou « texte ») est constitué de modifications non codées, de changements de genres, de changements de «mondes» ou encore de changements de places des locuteurs, ce que François résume sous le nom de « nouveauté ».

11 Dans un autre exemple (celui de la conduite de définition), l'efficacité du langage se traduit dans la capacité à reprendre le discours de l'autre ou à mettre en mots le réel, ou à faire un mouvement par rapport au discours de l'autre.

12 Au terme de cette deuxième partie, François préfère ainsi parler d'évolution plutôt que d'acquisition ou de développement du langage de l'enfant tant, selon lui, le langage évolue poussé par deux « moteurs » : l'activité même du sujet et la reprise-modification des discours de l'autre (« communauté » et « nouveauté »). Communication et évolution langagière sont étroitement corrélées. De même, il lui semble préférable de ne pas séparer a priori le rôle de l'organisation linguistique et le rôle des facteurs non linguistiques dans cette évolution, sous la forme de pratiques consistant, par exemple, à percevoir un objet de différents points de vue, à associer vue et toucher. C'est pourquoi les premières grammaires de l'enfant sont des grammaires où s'articule une signification portée soit par un mot et un geste à la fois (le pointage), soit par la relation entre deux mots. Les règles de grammaire ne se développent pas indépendamment des conditions d'utilisation du langage. En outre, l'enfant n'est jamais seul dans cette évolution: ses relations aux objets de son entourage sont médiatisées par l'adulte. Communiquer et manipuler sont sans cesse associés.

13 Étudier non la langue (c'est-à-dire les signes et leurs règles de combinaison) mais le langage (ce que l'enfant ou l'adulte fait avec le langage) en montrant, par exemple, tout ce l'on peut faire avec le même signe, telle est l'ambition de cet ouvrage où François souligne combien il est préférable d'étudier les conduites langagières plutôt que des unités et des structures linguistiques car l'enfant n'apprend pas directement des mots ou des phrases: il répète, répond, joue avec ce qu'il a appris. Dès lors mesurer des évolutions consistera à voir comment l'enfant parvient à prendre plus de distance par rapport à son énoncé, à augmenter le nombre de remarques critiques, de questions, de demandes d'explication, bref les progrès résident non seulement dans les moyens offerts par la langue mais aussi dans la reprise des « places » dialogiques et tout d'abord celles de l'adulte qui questionne, oriente la discussion, corrige, développe un thème... François étudie, à travers plusieurs exemples, la façon dont les énoncés se développent, 
soit en parallèle, soit en opposition, soit encore par coordination, par réaction finalement au discours de l'autre. Ainsi, dans le cas des conduites de définition étudiées p. 207 sq., l'enfant, s'il est seul face à l'adulte, isole bien souvent un seul aspect du phénomène considéré (« la tomate, c'est rouge »), alors que, face à un autre enfant, il parlera du même objet en en disant autre chose (« et puis ça pousse au soleil ») ou il parlera d'un autre objet en parallèle. Le signe-étiquette est acquis puis réutilisé dans un grand nombre de situations différentes. De même, l'oral s'étaye ou peut s'étayer sur la matérialité du corps, de la proximité, du contact, du métadiscours qu'est le regard qui enseigne ce que l'autre n'a pas compris.

La troisième partie de l'ouvrage est, sur certains points, davantage une unité autonome qu'une reprise-expansion des deux premières mais mérite d'être lue comme un éclairage supplémentaire des dénivellations dans le discours. Cette fois en effet, François entreprend d'étudier des échanges patient-médecin qui se concrétisent sous la forme d'enchaînements question-réponse-commentaire. Précisément, il étudie le discours pathologique à travers l'entretien psychiatre-patient ou à travers les œuvres de trois «psychotiques » : Schreber (et son commentaire par Freud), Perceval (étudié inauguralement par G. Bateson) et Rousseau. Qu'en est-il du langage dans le champ de ce qui est reconnu par ailleurs comme pathologie mentale? S'agit-il des mêmes mouvements discursifs que ceux précédemment analysés dans le champ de l'analyse du langage de l'enfant? L'ambition est d'emblée de mettre en doute la volonté unifiante du discours théorique (notamment psychanalytique) avec pour appui une linguistique $\mathrm{du}$ déroulement textuel, attentive à l'enchaînement des discours, plus qu'une linguistique des structures. Comment, par exemple, dans le cas d'un entretien psychiatre-patiente, est dite la souffrance? François étudie à la fois la place des interlocuteurs, les genres de discours différents qui sont au cœur de l'échange, avec comme objectif de mettre au jour les ressorts de l'efficacité du langage oral. Il propose de classer les dialogues médecin-malade sur un continuum qui va de l'entretien réglé à la conversation. Il n'y a pas, en effet, une réalité de l'obsession, un discours qui dirait cette réalité ; plutôt des modalisations monologiques, des mouvements par rapport au discours de l'autre et surtout des enchaînements thématiques. Comment encore, dans le cas des trois psychotiques, écrire sa folie: comment s'organise ici le discours? L'auteur met en évidence le fait que celui-ci se déploie tout d'abord par parenté et contraste avec d'autres discours (ceux de la religion, de la science, ceux des «amis »« ennemis » dans le cas de Rousseau). Il peut y avoir un autre mode d'interprétation du texte délirant qui consisterait à voir comment ces trois «psychotiques» montrent plusieurs façons d'exister par le discours dans leur relation à la folie. François étudie tout d'abord le discours de Schreber mais aussi et peut-être surtout la façon dont Freud a analysé ce discours en s'intéressant à des éléments du contenu, sélectionnés, et non au mode d'expression par le mélange discursif. Or ce mélange rend impossible la séparation entre l'expérience qu'il nous est donné de nous représenter de la façon dont l'auteur l'a mise en mots. À la différence de Perceval et de Rousseau, c'est moins ici un récit qui donne forme et valeur à des instants passés qu'une construction théorique argumentative.

15 François souligne le passage "réussi » de la névrose de Rousseau en auto-analyse ou quête de soi où se mettent en scène trois modalités discursives: le discours sur le paraître, le discours de la faute et celui de l'aveu. Le discours de Rousseau est une élaboration interprétante (ou une «interprétation persécutive») qui trouve à s'épancher dans le récit des Confessions ou des Rêveries du promeneur solitaire. Le 
mouvement de l'écriture donne sens et reprend le mouvement de la croyance. François décèle une forme de jeu dans le changement des modes de discours à l'œuvre dans ces formes d'écriture littéraire au point d'articulation de la preuve, du récit, du doute et de la description.

L'ouvrage présenté ici n'est sans doute pas celui qu'on citerait d'emblée pour souligner l'importance et la vivacité dérangeante de la pensée de F. François. Pourtant, lu par différence-rapprochement avec quelques autres (Pratiques de l'oral notamment), il confirme, s'il en était encore besoin, les enjeux de ces analyses du discours notamment dans le champ pédagogique. Il existe une zone intermédiaire où l'adulte et l'enfant comme le psychiatre et son patient peuvent expliciter ce qu'ils perçoivent, rendre compte de ce qu'ils disent tout en ayant un point de vue justifié : c'est la zone d'interprétation. Chaque enfant est ainsi quelque part entre l'interprète-lecteur générique et l'interprète-lecteur spécifique. L'interprétation n'est pas relation directe d'un discours à un état des choses mais reprise par l'enfant de discours déjà dits, ou opposition à ces discours, ou modification et ouverture du sens. Dans les cadres précédemment décrits, l'adulte propose à l'enfant de nouveaux scénarios qui sont des situations structurées où, ensemble, ils coopèrent. Nous voudrions trouver là un illustration de la prescription à " coopérer par le langage ", présente dans les dernières instructions officielles (BO, hors série, février 2002). La coopération se fera ici autant entre l'adulte et l'enfant qu'entre pairs. L'adulte est alors, dans ce cadre précis, moins un modèle qu'un partenaire dont le rôle est important car il aide l'enfant à clarifier ses intentions de communication, et à les ajuster aux conditions et aux exigences de la communauté. Ce moment constitue un lieu d'apprentissage authentique car il change pour l'enfant les conséquences des actions. L'enfant peut donc tenter des choses au plan langagier. Il s'agit alors pour lui, comme le souligne constamment François, d'un lieu d'expériences et d'expérimentations, à la fois de l'usage du langage, du rôle du contexte, de la communication et de l'autre comme interlocuteur. La construction d'un tel lieu nous invite à proposer un changement de paradigme grâce auquel on pourrait envisager les faits qui se présentent à nous dans le moment des échanges oraux en termes d'événement langagier, situé dans un cadre social, et moins en termes de développement ou même d'acquisition langagière. Ces deux derniers concepts suggèrent, en effet, soit un long processus déterminé surtout par des facteurs internes et communs pour l'essentiel à tous, soit un phénomène déterminé par des modèles reçus passivement. Il semble, par exemple, que le moment du récit et les échanges qu'il permet montrent, au contraire, qu'il y a bien ici association d'activités personnelles de l'enfant, reprises et intériorisation du discours de l'adulte dans des proportions qui dépendent non seulement de l'âge mais aussi de l'étayage apporté. Dans une telle circonstance, l'enfant apprend comment il peut contribuer, grâce au langage, à l'interaction sociale et à son dynamisme présent. Il apprend, pour cela, aussi bien les formes appropriées du discours que les modes sur lesquels le contenu de ses interventions, dans le cadre d'entretiens "métadiscursifs", doit être organisé afin d'être en rapport avec le contexte communicatif. L'authenticité et l'originalité que l'adulte reconnaitra à ses verbalisations contribuent fortement à faire de ces moments d'échanges un vecteur important de socialisation. 


\section{AUTEUR}

\section{CLAUDE LE MANCHEC}

Lidilem et INRP 